\title{
CrystEngComm
}

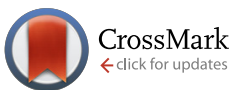

Cite this: CrystEngComm, 2014, 16, 9907

\section{Size control and shape evolution of single-twinned platinum nanocrystals in a room temperature colloidal synthesis $\uparrow$}

\author{
Milena Osmić, ${ }^{a}$ Joanna Kolny-Olesiak ${ }^{\star b}$ and Katharina Al-Shamery ${ }^{a}$
}

\begin{abstract}
Platinum nanocrystals in the size range of a few nanometers are especially interesting candidates for catalytic applications. We present an easy, low-temperature procedure, which allows for a precise size control of this material. Uniform platinum nanoparticles were obtained by reduction of platinum(iv)chloride with tetrabutylammonium borohydride in toluene solution at room temperature. Dodecylamine served as a ligand. The size of the resulting particles could be controlled in the range between 2 and $5 \mathrm{~nm}$ by slow addition of platinum monomers and the reducing agent to preformed $2 \mathrm{~nm}$ seeds. Furthermore, particles with a tetrapod shape could be synthesized. In contrast to the usual kinetic control leading to the formation of elongated structures, the analysis of the growth process of the tetrapods suggests a reactioncontrolled growth process. Their structure was investigated by powder X-ray diffraction and highresolution electron microscopy, which revealed that two branches of the tetrapods grow along the $\langle 111\rangle$ direction, while the other two show growth in the $\langle 220\rangle$ direction. This uncommon shape of the tetrapods is attributable to the presence of a twin defect parallel to the $\langle 220\rangle$ direction. Thus, the method presented in the article allows for the formation of platinum particles with a single twin defect with a high yield.
\end{abstract}

Received 1st July 2014,

Accepted 4th September 2014

DOI: $10.1039 / c 4 c e 01342 e$

www.rsc.org/crystengcomm and do not require complicated experimental setups, expensive chemicals and high energy consumption (e.g. because of the need for high temperature) during the synthesis. In many studies published to date, conditions such as argon atmosphere and high temperatures were necessary to obtain size and shape control. ${ }^{15}$ Furthermore, relatively expensive educts, such as platinum acetylacetonate $\left(\mathrm{Pt}(\mathrm{acac})_{2}\right),{ }^{19,21,31-33}$ were used as metal precursors, and high purity of the educts and stabilizing agents was required. In some reactions, the size and shape control was achieved by the addition of compounds such as iron pentacarbonyl ${ }^{33}$ or silver nitrate. ${ }^{6} \mathrm{~A}$ drawback of these methods, however, is the possibility of alloy formation, which could influence the catalytic performance of the resulting particles. Another strategy uses peptides, which were found to bind specifically to certain facets of platinum nanocrystals, leading to the formation of tetrapods or facetted crystals. ${ }^{20,34}$

Small platinum nanoparticles with diameters of about 2 $\mathrm{nm}$ can be easily synthesized in organic solution at room temperature and under atmospheric pressure by reduction of platinum(Iv)chloride with tetrabutylammonium borohydride with dodecylamine as a ligand. ${ }^{35}$ By modification of this synthetic method, $2 \mathrm{~nm}$ thick nanowires, which grow by oriented attachment of quasi-spherical $2 \mathrm{~nm}$ particles, can be obtained..$^{30}$ Furthermore, variations of this synthesis can be applied to synthesize bimetallic platinum-tin particles. ${ }^{36}$ However, the possibilities of influencing the size of the 
particles synthesized with this method are rather limited, and attempts to grow larger particles by increasing the concentration of the precursors or by instantaneous injection of additional monomers to preformed particles do not lead to the expected outcome but result, e.g., in the formation of nanowires or the formation of additional small particles. ${ }^{30}$

Here, we present a method to control the size of platinum nanoparticles without the need for additional chemicals or elevated temperature. We use $2 \mathrm{~nm}$ Pt nanocrystals as seeds and overgrow them with further layers of platinum atoms by a controlled addition of platinum monomers and a reducing agent, using a syringe pump. We studied the impact of the reaction parameters on the size and the shape of Pt nanocrystals and found the optimum conditions for controlling the size of the resulting particles by adjusting the amount of added monomers in a reliable and predictable way. The particles were characterized by transmission electron microscopy (TEM) and X-ray diffraction (XRD) measurements. Furthermore, the analysis of high-resolution TEM images gave us insight into the shape evolution of the nanocrystals.

\section{Experimental}

\section{Materials}

Platinum(Iv)chloride $\left(\mathrm{PtCl}_{4}, 99 \%\right)$ was purchased from Acros Organics, dodecylamine (DDA, 98\%), tetrabutylammonium borohydride (TBAB, 98\%) and didodecyldimethylammonium bromide (DDAB, 98\%) from Aldrich and toluene (99.7\%) from VWR BDH Prolabo. All chemicals were used without further purification.

\section{Synthesis of $2 \mathrm{~nm}$ platinum seeds}

$186.0 \mathrm{mg}(0.906 \mathrm{mmol})$ of DDA was dissolved in $2.5 \mathrm{ml}$ of toluene. Then, $8.5 \mathrm{mg}(0.025 \mathrm{mmol})$ of $\mathrm{PtCl}_{4}$ was added and sonicated to obtain an optically clear yellow solution. Next, $25.0 \mathrm{mg}(0.097 \mathrm{mmol})$ of ТВAB and $46.3 \mathrm{mg}(0.100 \mathrm{mmol})$ of $\mathrm{DDAB}$ were dissolved in $1.0 \mathrm{ml}$ of toluene. To start the reaction, the TBAB/DDAB solution was rapidly injected into the $\mathrm{PtCl}_{4} / \mathrm{DDA}$ solution while stirring. The color of the solution turned black after one minute. The formation of the seeds was completed after 30 minutes.

The product solution $(3.5 \mathrm{ml})$ was purified by the addition of $14 \mathrm{ml}$ of methanol and centrifuged for 10 minutes to separate the Pt particles from the solvent. Next, the Pt particles were dissolved in $2 \mathrm{ml}$ of hexane. $8 \mathrm{ml}$ of methanol and $16 \mathrm{ml}$ of acetone were added to $2 \mathrm{ml}$ of this solution; subsequently, this mixture was centrifuged for 10 minutes. The precipitate containing the Pt particles was dissolved in $2 \mathrm{ml}$ of hexane.

\section{Synthesis of 3.5-6 nm Pt nanocrystals}

$1 \mathrm{ml}$ of the solution of the seeds was diluted with $50 \mathrm{ml}$ of toluene. Subsequently, $\mathrm{PtCl}_{4} / \mathrm{DDA}$ and DDAB/TBAB solutions were slowly injected into this seed solution using a KDS Scientific injection pump at a speed rate of $0.1 \mathrm{ml} \mathrm{min}^{-1}$. Table 1
Table 1 Reaction parameters for various sizes of Pt nanoparticles

\begin{tabular}{llllll}
\hline Theoretical size $^{a}$ & $2.0 \mathrm{~nm}$ & $3.5 \mathrm{~nm}$ & $4.0 \mathrm{~nm}$ & $5.0 \mathrm{~nm}$ & $6.0 \mathrm{~nm}$ \\
\hline $\begin{array}{l}\text { Expected volume increase } \\
\text { Solution 1 }\end{array}$ & & $5.4 \times$ & $8.0 \times$ & $15.6 \times$ & $27.0 \times$ \\
$\begin{array}{l}\text { PtCl } 4 \text { [mmol] } \\
\text { DDA [mmol] }\end{array}$ & 0.085 & 0.100 & 0.202 & 0.379 & 0.681 \\
Toluene [ml] & 1.004 & 4.014 & 4.417 & 15.053 & 25.088 \\
Solution 2 & 2.5 & 5.0 & 10.0 & 19.0 & 34.0 \\
$\begin{array}{l}\text { DDAB [mmol] } \\
\text { TBAB [mmol] }\end{array}$ & 0.100 & 0.389 & 0.777 & 1.457 & 2.623 \\
Toluene [ml] & 0.097 & 0.400 & 0.801 & 1.501 & 2.702 \\
a & 1.0 & 5.0 & 10.0 & 19.0 & 34.0 \\
Final size of the nanocrystals, calculated & assuming & $100 \%$ \\
conversion of the monomers. & & & &
\end{tabular}

shows an overview of the amounts of the chemicals used in reactions leading to different sizes of the nanoparticles. The particles were subsequently cleaned using the same procedure as in the case of the $2 \mathrm{~nm}$ seeds.

\section{Characterization}

TEM images of the Pt particle were taken with a Zeiss EM 902 electron microscope. $6.7 \mu$ l of the diluted solution was dropped on a carbon-coated copper grid and dried at room temperature. HRTEM images were obtained with a JEOL JEM2100F microscope, operating at $200 \mathrm{kV}$.

XRD was measured with a PANalytical X'Pert PRO MPD diffractometer operating with $\mathrm{Cu} \mathrm{K} \alpha$ radiation, Bragg-Brentano $\theta-2 \theta$ geometry, and a goniometer radius of $240 \mathrm{~mm}$. Samples for XRD measurement were prepared by drying the purified Pt nanocrystals solution on low background silicon substrates.

\section{Results and discussion}

Size control of Pt nanocrystals obtained within this study builds on the heterogeneous nucleation of platinum atoms on the surface of preformed $2 \mathrm{~nm}$ seed particles. Therefore, to obtain a narrow size distribution of the resulting particles, it is essential to avoid homogeneous nucleation parallel to the growth. The generation of new, small particles would broaden the size distribution. Thus, conditions have to be evaluated, under which the rate of homogeneous nucleation is negligible.

The parameters which influence the formation of new seeds are temperature and the concentration of the monomers (or, more precisely, the oversaturation of the solution). Thus, homogeneous nucleation can be suppressed by lowering the temperature or the concentration of the reaction solution. To find the optimum platinum concentration of the reaction solution, the volume of the solvent was increased while the amount of all the other chemicals was kept constant, which resulted in dilution factors in the range between 2.5 and 125 . In reactions with 100 and $125 \mathrm{ml}$ of toluene, which correspond to platinum concentrations of $8.5 \times 10^{-4}$ mmol ml$l^{-1}$ and $6.8 \times 10^{-4} \mathrm{mmol} \mathrm{ml}^{-1}$, respectively, no formation of platinum nanocrystals could be observed. Under these reaction conditions, the concentration of the educts 
apparently falls below the threshold of homogenous nucleation. Thus, in order to favor heterogeneous nucleation, the concentration of the monomers should not exceed $8.5 \times 10^{-4}$ mmol ml $\mathrm{m}^{-1}$ during the addition of the monomers to the solution of the seeds.

The reduction of platinum ions and the growth of platinum particles are relatively fast processes; therefore, in a solution containing platinum seeds a substantial fraction of the monomers should be used up within several minutes from their injection. Thus, we should be able to reach a tiny enough concentration of the monomers without the need of diluting the solution of the seeds as much as 100 times. Therefore, we tested what happens if all the monomers are introduced at once to the 50 times diluted solution of the seed particles. The resulting nanocrystals were short nanowires; thus, if we just want to increase the size of the particles, a higher dilution or a slower addition of the monomers is necessary. To evaluate the optimum values for both parameters, we tested three injection rates $(0.08,0.1$ and $0.2 \mathrm{ml}$ per $\mathrm{min})$, trying also different dilutions of the seed solution $(1: 25$, $1: 50,1: 75$ and $1: 100)$; see Table 2 and Fig. S1 in the ESI. $\dagger$ We compared the size, shape and size distribution of the particles resulting from different combinations of both parameters. The anticipated size of the particles was $4 \mathrm{~nm}$. In all 12 reactions, the obtained diameters are close to this value; however, there are differences in the size distribution and the shape of the particles. Not all the samples consisted of spherical particles; some of the nanocrystals had irregular or facetted shapes. Optimum results (quasi-spherical particles with a narrow size distribution and a diameter of $3.95 \pm$ $0.42 \mathrm{~nm}$ ) were obtained with an injection rate of $0.1 \mathrm{ml} \mathrm{min} \mathrm{m}^{-1}$ and a dilution of $1: 50$. Therefore, these parameters were used for further experiments.

Fig. 1 shows overview TEM images of particles obtained in experiments aiming at size control of the nanoparticles. To achieve this goal, different amounts of platinum monomers and reducing agent were added slowly to the seed solution. The amounts were calculated considering the increase of the volume from $2 \mathrm{~nm}$ to the target size, assuming a conversion of $100 \%$. The size of the nanocrystals was varied between 2 and $6 \mathrm{~nm}$ (see Fig. 1a-e) and their size distribution is relatively narrow, with standard deviations between $11 \%$ and

Table 2 Sizes of particles obtained in reactions with different injection rates and dilution of the seed solution. The targeted size of the nanocrystals was $4 \mathrm{~nm}$

\begin{tabular}{|c|c|c|c|}
\hline \multirow[b]{3}{*}{ Dilution } & \multicolumn{3}{|c|}{ Injection rate $\left[\mathrm{ml} \mathrm{min}^{-1}\right]$} \\
\hline & 0.08 & 0.1 & 0.2 \\
\hline & Size $[\mathrm{nm}]$ & & \\
\hline $1: 25$ & $4.53 \pm 0.47^{a}$ & $5.28 \pm 0.60^{a}$ & $3.82 \pm 0.54$ \\
\hline $1: 50$ & $3.83 \pm 0.60$ & $3.95 \pm 0.42$ & $4.47 \pm 0.58^{a}$ \\
\hline $1: 75$ & $3.09 \pm 0.53$ & $3.76 \pm 0.58$ & $3.52 \pm 0.51$ \\
\hline $1: 100$ & $4.27 \pm 0.53^{a}$ & $3.74 \pm 0.40$ & $3.79 \pm 0.50$ \\
\hline
\end{tabular}

${ }^{a}$ Particles formed under these conditions had facetted or branched shapes, which leads to a larger outer diameter of the nanocrystals compared with the anticipated size of $4 \mathrm{~nm}$.
$15 \%(1.91 \pm 0.27 \mathrm{~nm}, 3.30 \pm 0.39 \mathrm{~nm}, 3.90 \pm 0.47 \mathrm{~nm}, 5.25 \pm$ $0.57 \mathrm{~nm}$, and $5.75 \pm 0.87 \mathrm{~nm})$.

A closer look at the TEM images reveals changes in the shape of the particles during their growth process. While 2-4 nm particles exhibit quasi-spherical shapes (Fig. 1a-c), 5 and $6 \mathrm{~nm}$ particles are facetted, mostly with rectangular or triangular projection in the TEM image (Fig. 1d and e), which suggests that they are terminated by low indexed, e.g., $\{100\}$ and $\{111\}$, surfaces. Also, a slight tendency to form elongated or branched structures is apparent. Furthermore, in the case of the $6 \mathrm{~nm}$ particles, we observe a ripening process leading to the formation of tetrapods (Fig. 1f) when the particles are left overnight at room temperature.

The structure and the size of the particles were further investigated with XRD measurements. Fig. 2 shows the XRD patterns of the different samples. The reflections are broadened because of the small size of crystallites, but their positions fit well the values for the fcc-Pt structure. The reflections were fitted with Gaussian functions to extract the value of their full width at half maximum (FWHM), which is needed to calculate the size of the particles using the Scherrer formula (here used with $K=1$ for undefined geometry). The peaks in the XRD patterns of quasi-spherical particles could be accurately fitted with single Gaussians. The results match well with the values obtained from the analysis of the TEM images, as can be seen in Fig. 3, which compares the anticipated values with the ones obtained from TEM and XRD. Also the size of the particles measured in the TEM images corresponds well to the targeted size. Thus, our method allows for precise size control of platinum nanocrystals by simply adjusting the amount of platinum monomers overgrowing the seed particles.

To gain insight into the structural properties of the synthesized nanocrystals and their growth process, we compared the diffraction patterns of the $6 \mathrm{~nm}$ particles and the branched nanocrystals obtained during their ripening process. In the diffraction pattern of the $6 \mathrm{~nm}$ particles, the 200 and 220 reflections could be again fitted with single Gaussians and yield crystallite sizes of 4.8 and $8.6 \mathrm{~nm}$ along the $\langle 200\rangle$ and $\langle 220\rangle$ directions. In contrast to this, the line shape of the 111 reflection has to be fitted with two Gaussians of different widths and relative intensities of $83: 17$, corresponding to 4.1 and $8.1 \mathrm{~nm}$, respectively (see the green lines in the XRD pattern of $6 \mathrm{~nm}$ in Fig. 2). For perfectly spherical particles, the crystallite sizes obtained from different reflections should not differ from one another. However, the presence of facetted, e.g. cubic, particles in the sample could explain the discrepancy between the crystallite sizes along the $\langle 200\rangle$ and $\langle 220\rangle$ directions. For a cube, the crystallite size does not differ along all the six possible $\langle 220\rangle$ directions ([220], [202], [022], [-220], [-202], and [02-2]). Therefore, the line shape of the 220 reflection should be a single Gaussian, with a FWHM corresponding to the length of the surface diagonal of the cube. The length of the surface diagonal of a $5 \mathrm{~nm}$ cube is $\sim 7 \mathrm{~nm}$, which is in good agreement with the obtained results. A similar geometrical consideration yields a crystallite 


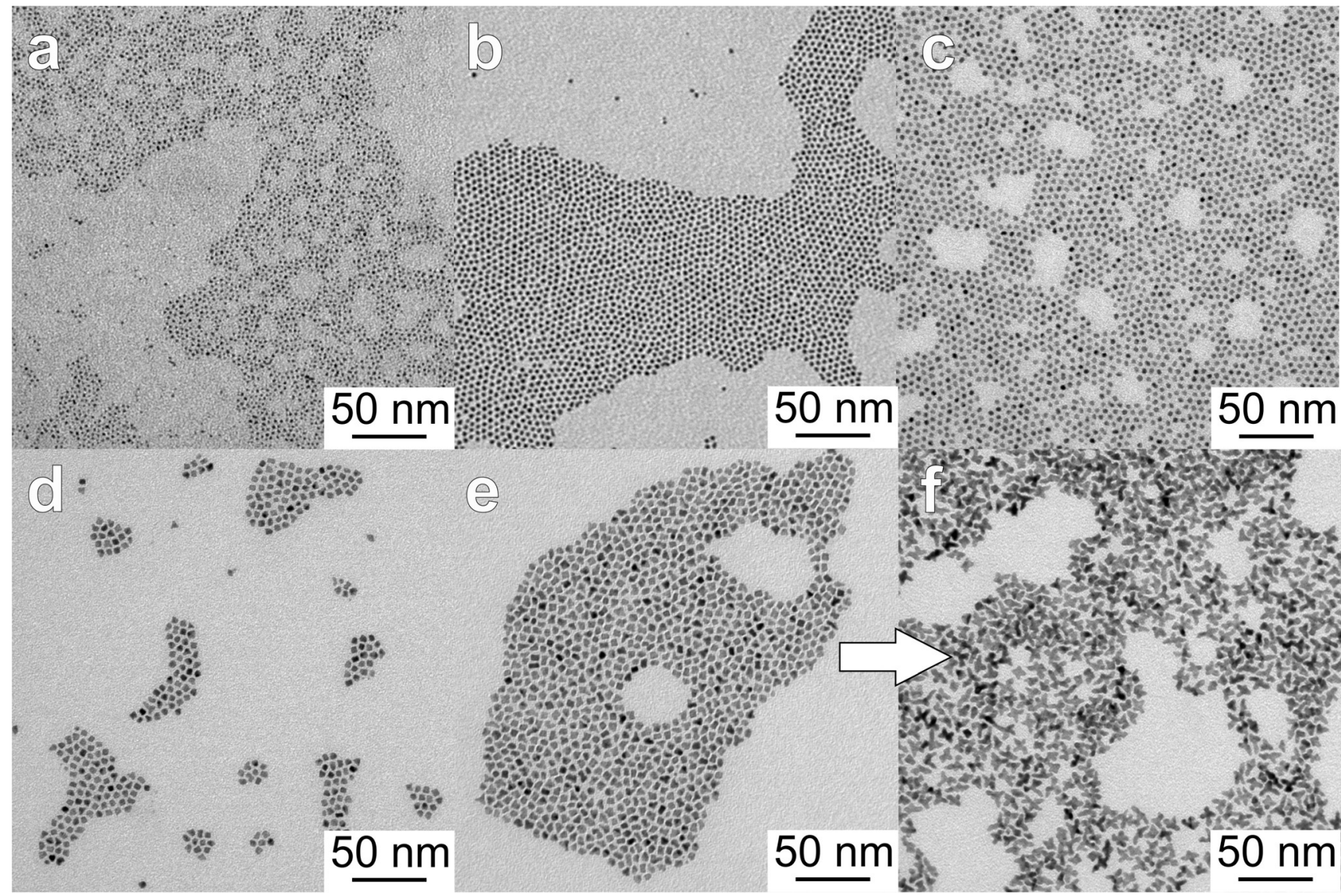

Fig. 1 Overview TEM images of quasi-spherical platinum nanocrystals: a) $2 \mathrm{~nm}$, b) $3.5 \mathrm{~nm}$, c) $4 \mathrm{~nm}$, d) $5 \mathrm{~nm}$, e) $6 \mathrm{~nm}$ and f) multipods obtained during a ripening process of the $6 \mathrm{~nm}$ particles in d).

size of $8.6 \mathrm{~nm}$ along the $\langle 111\rangle$ direction (again for a $5 \mathrm{~nm}$ cube). However, a cubic shape does not account for the presence of two components with different widths in the 111 reflection. This can only be explained with a preferential growth along the $\langle 111\rangle$ direction (see below for a detailed explanation). Thus, the analysis of the XRD pattern of the $6 \mathrm{~nm}$ sample shows contributions from different shapes of the nanoparticles. Furthermore, a tendency to preferential growth along the $\langle 111\rangle$ direction can be observed. These findings are consistent with the observations from TEM, showing some facetted and some particles developing short branches.

While in the diffraction pattern of the tetrapods the 200 reflection could again be fitted with single Gaussians (corresponding to a crystallite size of $6.3 \mathrm{~nm}$ ), the line shapes of the 111 and 220 reflections significantly differ from that of a single Gaussian. However, they could be fitted accurately using two Gaussians with different widths (see the green lines in the upper panel of Fig. 2). The sizes of the crystallites obtained from the FWHM of both contributions to the 111 reflection are 4.4 and $10.3 \mathrm{~nm}$, while the evaluation of the FWHM of the 220 reflection yields 7.1 and $16.5 \mathrm{~nm}$. The relative intensities of the peaks corresponding to the smaller and larger crystallite sizes are $73: 27$ and $72: 28$ for the 111 and 220 reflections, respectively.
This bimodal size distribution with respect to one growth direction of the crystallites can be understood, when considering the symmetry of the fcc lattice. A nanorod, which grows along the [111] direction, is spatially limited along the [11-1], $[1-11]$ and $[-111]$ directions, which all give rise to a reflection at the same angle in the powder diffraction pattern. Therefore, for this kind of nanorod, we would expect a 111 reflection which is an overlap of a broad and a narrow component, corresponding to the width and the length of the nanorod, respectively, with an intensity ratio of $75: 25$. Analog consideration can be done for a nanorod growing along the $\langle 220\rangle$ direction. Therefore, the analysis of the line profile of the 111 reflection and of the intensity of both contributions to the 111 reflection is in line with the assumption that the tetrapods grow in the $\langle 111\rangle$ direction. However, from the analysis of the 220 reflection, a growth along the $\langle 220\rangle$ direction can be deduced.

It is surprising at first glance to find two preferential growth directions for the tetrapods forming in our reaction. One explanation could be the formation of a mixture of two kinds of particles, one growing along the $\langle 111\rangle$ direction and the other along the $\langle 220\rangle$ direction. However, we would not expect in this case to find intensity ratios so close to $75: 25$ for the broader and the narrower Gaussians. Another possibility 


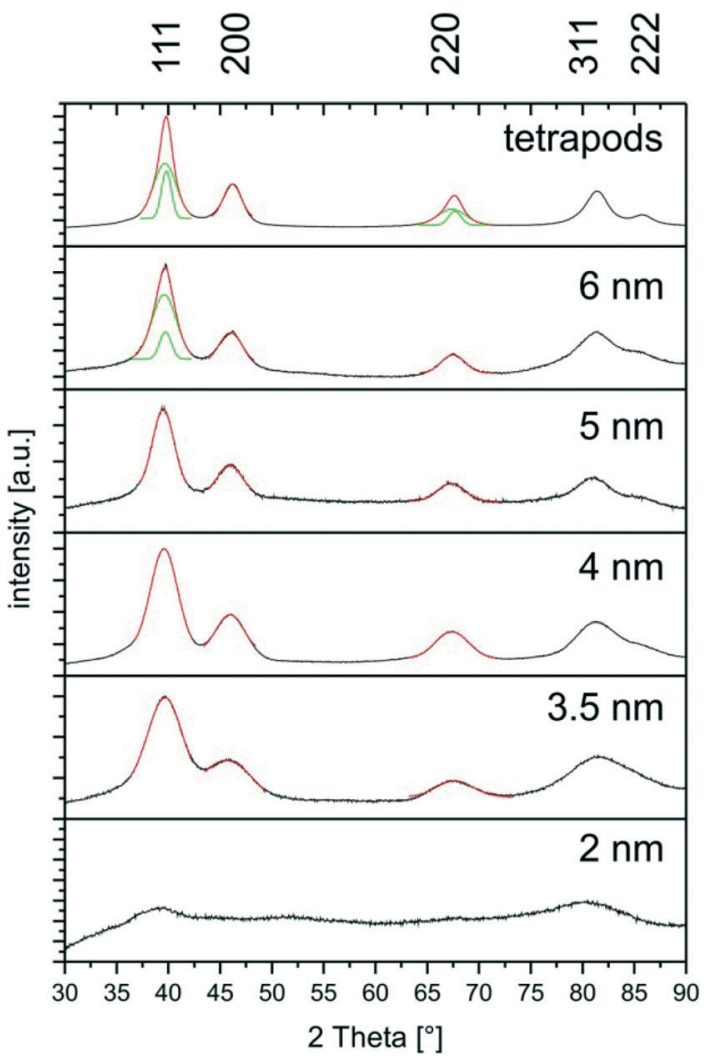

Fig. 2 XRD patterns of Pt nanocrystals with different sizes. 111, 200 and 220 reflections were fitted using Gaussians. The fitting results are shown in red. If more than one Gaussian was necessary to fit a reflection, the components are presented in green.

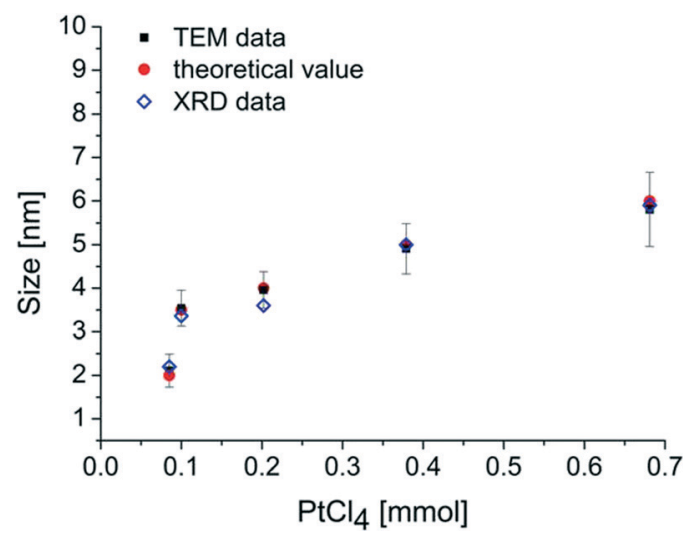

Fig. 3 Size of nanoparticles obtained from different amounts of platinum monomers. Experimental results obtained with TEM and XRD are compared to theoretical sizes. For the evaluation of the TEM images, 300 particles on average were measured for each size. The size obtained from the XRD data is an average number obtained from the evaluation of the broadening of the 111, 200 and 220 reflections. The theoretical size is the diameter of the particles calculated assuming $100 \%$ conversion of the added monomers.

is the growth of tetrapods with two kinds of branches, some growing along the $\langle 111\rangle$ direction and some along the $\langle 220\rangle$ direction. The analysis of high-resolution TEM images (see below) shows that this is the case.
The size and the shape evolution of the particles during the addition of monomers and their ripening process give us some hints about their growth process, as will be discussed in the subsequent paragraphs.

Particles with sizes between 2 and $4 \mathrm{~nm}$ grow only during the addition of the monomers and no subsequent ripening process or shape change was observed. This shows that all the added monomers reacted with the existing particles immediately after the injection. Thus, for this size range $(2-4 \mathrm{~nm})$ and at the injection rate chosen for our experiments $\left(0.1 \mathrm{ml} \mathrm{min}^{-1}\right)$, the transport of the monomers to the surface of the particles was the major factor limiting the growth rate of the nanocrystals, while the rate of the reaction of the monomers with the surface of the nanoparticles played a secondary role. This reaction can be assumed to be relatively fast, owing to the high surface energy of the small quasi-spherical particles.

In contrast to experiments resulting in the formation of quasi-spherical particles, the reaction which leads to the formation of $6 \mathrm{~nm}$ particles needs more time to be completed. This is, at first, an unexpected observation, which shows that the growth mechanism probably changes, while the particles grow larger. Although the injection rate does not change and the overall surface of the particles increases with increasing diameter, not all the monomers react immediately with particles in the solution. For particles terminated by low indexed facets, which are less reactive, compared to quasi-spherical ones, the reaction rate on the surface seems to be the growth-limiting factor. The shape evolution of the $6 \mathrm{~nm}$ particles gives us additional insight into the reasons for this behavior. Their growth does not finish immediately after the addition of the monomers but continues for several hours afterwards. Fig. 1 shows the particles one hour after the end of the addition of the monomers (Fig. 1e) and after ripening overnight (Fig. 1f). The growth process does not occur on the whole surface of the particles but is limited to the most reactive facets of the nanocrystals, which results in anisotropic growth and the formation of tetrapods. Thus, in contrast to the kinetically controlled growth, which is usually the reason for the formation of elongated or branched structures, ${ }^{5,19,26}$ the availability of reactive regions on the surface of the particles accounts for the tetrapod formation in our reaction.

In addition, the temperature is a crucial parameter influencing the growth kinetics; therefore, we studied its impact on the growth of the particles. The $6 \mathrm{~nm}$ particles were chosen for this purpose because of the possibility to observe the role of temperature not only in controlling the size but also the shape of the resulting nanoparticles. A small increase in temperature (from 18 to $24{ }^{\circ} \mathrm{C}$ ) has an influence on the resulting size and shape of the nanocrystals; the particles are facetted or have slightly irregular shapes, but they do not form branches (Fig. 4b). Furthermore, they do not reach the anticipated size of $6 \mathrm{~nm}$, which suggests that the monomers are partly used up for the formation of additional seeds. This is even more pronounced when the temperature is increased further to 50 or $100{ }^{\circ} \mathrm{C}$ (Fig. $4 \mathrm{~d}$ and f). The size 


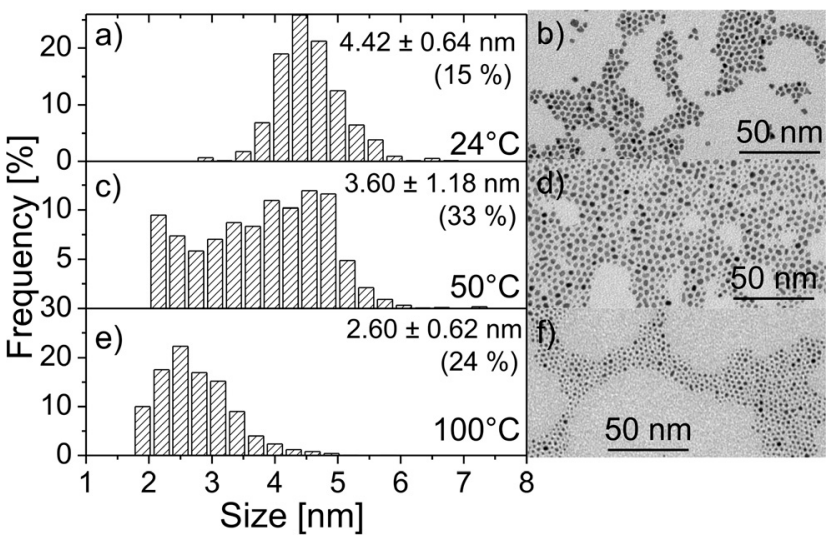

Fig. 4 Size histograms of particles synthesized at different temperatures $(a, c$, and e) together with the overview TEM images (b, $d$, and f). The targeted size was $6 \mathrm{~nm}$.

distribution of the particles broadens, while their size decreases (Fig. 4a, c, and e). The size distribution histogram of the particles grown at $50^{\circ} \mathrm{C}$ shows (in outlines) a bimodal size distribution, with a substantial fraction of newly formed particles together with larger particles obtained by the growth of the original seeds. Thus, at this temperature both nucleation and growth take place in the reaction solution. At $100{ }^{\circ} \mathrm{C}$, the nucleation outweighs the growth of the existing seeds, which results in a rather broad size distribution and formation of nanoparticles substantially smaller than the anticipated size. These experiments show that under our reaction conditions homogeneous nucleation can only be avoided effectively at room temperature and below. Therefore, increasing the reaction temperature is not a suitable method to influence the size or the shape of the resulting nanocrystals.

To get further insight into the growth process of platinum particles and their shape control, the samples were characterized with high-resolution TEM. Representative images for all the samples of particles with different sizes can be found in Fig. 5. Most of the particles seem to be single crystalline; however, a closer view of the micrographs reveals that particles with twin defects can be found in all the samples from 3.5 to $6 \mathrm{~nm}$ (see also examples in Fig. 6a and b).

A single twin defect can be detected only in some specific orientations of the particle towards the electron beam, while the particle can appear single crystalline in other orientations. For instance, a twin defect (in our example, a (1-11) mirror plane), which is visible in [011] orientation (Fig. 6d), is not detectable in a particle which was rotated $90^{\circ}$ around the $b$-axis (in [0-11] orientation, Fig. 6e). Therefore, from the analysis of the HRTEM images of the quasi-spherical particles, which do not exhibit any preferred orientation on the TEM grid, we cannot draw conclusions about the fraction of

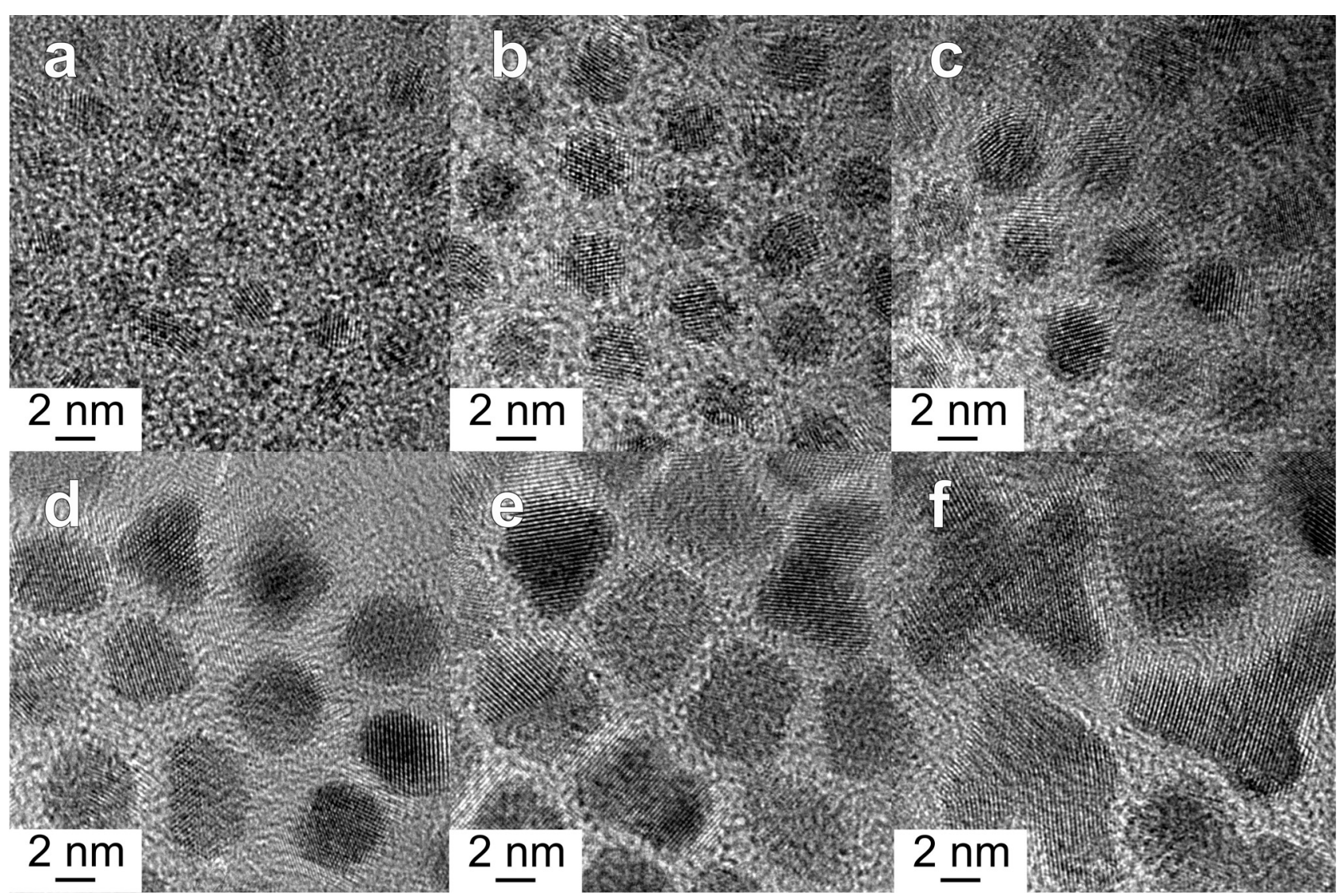

Fig. 5 HRTEM images of quasi-spherical platinum nanocrystals: a) $2 \mathrm{~nm}$, b) $3.5 \mathrm{~nm}$, c) $4 \mathrm{~nm}$, d) $5 \mathrm{~nm}$, e) $6 \mathrm{~nm}$ and f) multipods obtained during a ripening process of the $6 \mathrm{~nm}$ particles in d). 


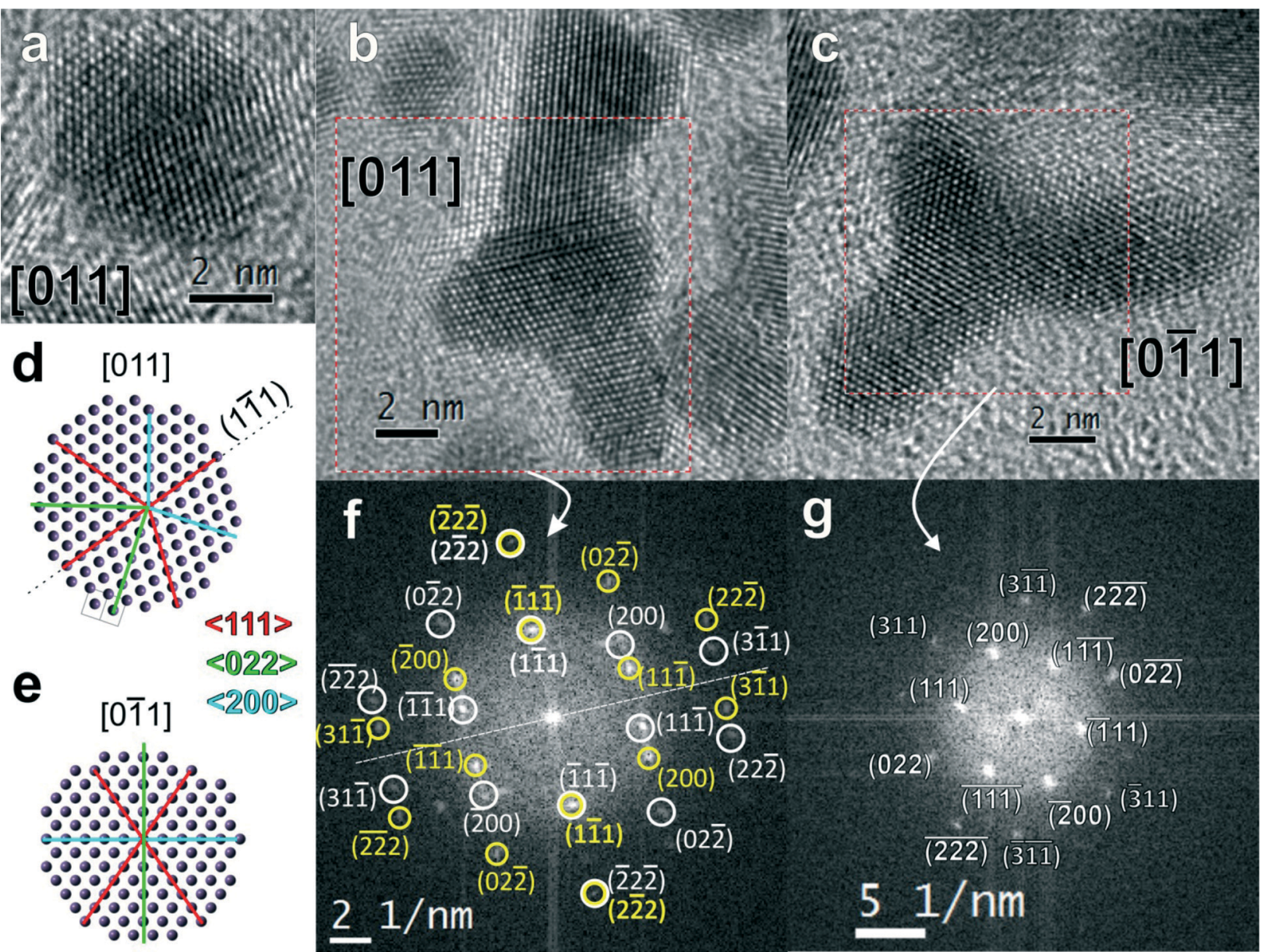

Fig. 6 HRTEM images of a $5 \mathrm{~nm}$ particle with a twin plane (a) and tetrapods ( $b$ and c) in two different orientations to the electron beam: [011] (b) and [0-11] (c). Panels (d) and (e) show a model of a platinum nanoparticle with a (1-11) twin plane (indicated by the dashed line) in [011] (d) and [0-11] (e) orientations. $\langle 111\rangle,\langle 022\rangle$ and $\langle 200\rangle$ lattice planes are marked with red, green and blue lines. Indexed FTT patterns of the particles from (b) and (c) are presented in ( $f$ ) and (g), respectively. The region of the image taken for the calculation of the FFT is indicated by the red square. In (f), the presence of a mirror plane is indicated by the dashed line and the patterns corresponding to the upper and lower parts of the particle are indexed using white and yellow colour, respectively.

defect-free particles within our samples. In contrast to silver and gold, there are not many reports of controlled growth of twinned platinum nanocrystals. The twin formation energy is higher for Pt than Ag, Au or Pd; therefore, twinned Pt structures are generally less frequently observed. In our reaction, we have only an indirect indication of the formation of twinned nanocrystal with a high yield, which is based on the shape evolution of our nanoparticles. During the ripening process of the $6 \mathrm{~nm}$ particles, tetrapods are formed, which however do not exhibit the tetrahedral symmetry, which would be anticipated for single crystalline structures.

The presence of a twin plane leads to a peculiar shape of the tetrapods, which is composed of a nanorod growing in the $\langle 011\rangle$ direction with two branches growing in the $\langle 111\rangle$ direction. The branches do not start exactly in the middle of the central rod, which leads to the formation of an asymmetric structure, with two "branches" (parts of the central "nanorod") growing along the same direction but with different lengths (Fig. 6b). The projection of this shape along the growth direction of the central nanorod [0-11] looks like a single crystalline tripod (see Fig. 6c). This analysis of HRTEM images confirms our conclusions drawn from the evaluation of the line profiles in the XRD patterns, which suggested two preferential growth directions for each tetrapod.

\section{Conclusions}

A slow injection of additional monomers into a solution of $2 \mathrm{~nm}$ platinum seeds is a suitable way to control the size of the particles in a precise way. The exact amount of monomers needed to obtain a specified size can simply be calculated from the anticipated increase in the volume of the particles. This method can be applied to obtain particles in the size range between 2 and $6 \mathrm{~nm}$, which is especially interesting for catalytic applications of these materials.

Not only quasi-spherical but also facetted and branched nanocrystals could be obtained with our method. At a size of $\sim 4 \mathrm{~nm}$, we observe a transition between quasi-spherical and facetted crystals, which noticeably slows down their further growth rate and induces the formation of branched 
structures instead of larger quasi-spherical or facetted particles. Owing to the low reactivity of the low indexed facets, the growth does not take place on the whole surface of the nanoparticles but only at some specific places with the highest curvature and, consequently, lowest coverage by the ligand molecules. An analysis of the shape and the crystallographic structure of the tetrapods shows that their specific shape is determined by the presence of a twin defect.

\section{Acknowledgements}

We gratefully acknowledge funding of the EWE Research Group "Thin Film Photovoltaics" and of the project "Nanostructured Fischer-Tropsch Catalysts Systems: Catalysts for the Generation of Synthetic Fuel from Biomass" by the EWE AG, Oldenburg.

\section{Notes and references}

1 M. De, P. Ghosh and V. Rotello, Adv. Mater., 2008, 20, 4225-4241.

2 Y. Tang and W. Cheng, Sci. Adv. Mater., 2012, 4, 784-797.

3 B. F. G. Johnson, Top. Catal., 2003, 24, 147-159.

4 B. R. Cuenya, Thin Solid Films, 2010, 518, 3127-3150.

5 H. Lee, S. E. Habas, S. Kweskin, D. Butcher, G. A. Somorjai and P. Yang, Angew. Chem., Int. Ed., 2006, 45, 7824-7828.

6 M. E. Grass, Y. Yue, S. E. Habas, R. M. Rioux, C. I. Teall, P. Yang and G. A. Somorjai, J. Phys. Chem. C, 2008, 112, 4797-4804.

7 R. Narayanan and M. A. El-Sayed, Nano Lett., 2004, 4, 1343-1348.

8 H. Borchert, D. Fenske, J. Kolny-Olesiak, J. Parisi, K. Al-Shamery and M. Bäumer, Angew. Chem., Int. Ed., 2007, 46, 2923-2926.

9 D. Fenske, P. Sonström, J. Stöver, X. Wang, H. Borchert, J. Parisi, J. Kolny-Olesiak, M. Bäumer and K. Al-Shamery, ChemCatChem, 2010, 2, 198-205.

10 A. Chen and P. Holt-Hindle, Chem. Rev., 2010, 110, 3767-3804.

11 J. Park, J. Joo, S. G. G. Kwon, Y. Jang and T. Hyeon, Angew. Chem., Int. Ed., 2007, 46, 4630-4660.

12 A. R. Tao, S. Habas and P. Yang, Small, 2008, 4, 310-325.

13 W. Zhu, A.-X. Yin, Y.-W. Zhang and C.-H. Yan, Chem. - Eur. J., 2012, 18, 12222-12226.

14 X. Zhong, Y. Feng, I. Lieberwirth and W. Knoll, Chem. Mater., 2006, 18, 2468-2471.
15 H.-T. Zhang, J. Ding and G.-M. Chow, Langmuir, 2008, 24, 375-378.

16 T. Yao, S. Liu, Z. Sun, Y. Li, S. He, H. Cheng, Y. Xie, Q. Liu, Y. Jiang, Z. Wu, Z. Pan, W. Yan and S. Wei, J. Am. Chem. Soc., 2012, 134, 9410-9416.

17 Z. L. Wang, J. M. Petroski, T. C. Green and M. A. El-Sayed, J. Phys. Chem. B, 1998, 102, 6145-6151.

18 N. Tian, Z.-Y. Zhou, S.-G. Sun, Y. Ding and Z. L. Wang, Science, 2007, 316, 732-735.

19 X. Teng and H. Yang, Nano Lett., 2005, 5, 885-891.

20 L. Ruan, C.-Y. Chiu, Y. Li and Y. Huang, Nano Lett., 2011, 11, 3040-3046.

21 J. Ren and R. D. Tilley, Small, 2007, 3, 1508-1512.

22 E. Ramirez, L. Eradès, K. Philippot, P. Lecante and B. Chaudret, Adv. Funct. Mater., 2007, 17, 2219-2228.

23 M. N. Mankin, V. Mazumder and S. Sun, Chem. Mater., 2011, 23, 132-136.

24 S. Maksimuk, X. Teng and H. Yang, Phys. Chem. Chem. Phys., 2006, 8, 4660-4663.

25 S. Maksimuk, X. Teng and H. Yang, J. Phys. Chem. C, 2007, 111, 14312-14319.

26 L.-M. Lacroix, C. Gatel, R. Arenal, C. Garcia, S. Lachaize, T. Blon, B. Warot-Fonrose, E. Snoeck, B. Chaudret and G. Viau, Angew. Chem., Int. Ed., 2012, 51, 4690-4694.

27 M. R. Axet, K. Philippot, B. Chaudret, M. Cabié, S. Giorgio and C. R. Henry, Small, 2011, 7, 235-241.

28 J. Chen, T. Herricks, M. Geissler and Y. Xia, J. Am. Chem. Soc., 2004, 126, 10854-10855.

29 J. Chen, T. Herricks and Y. Xia, Angew. Chem., Int. Ed., 2005, 44, 2589-2592.

30 D. Fenske, H. Borchert, J. Kehres, R. Kröger, J. Parisi and J. Kolny-Olesiak, Langmuir, 2008, 24, 9011-9016.

31 S. Cheong, J. Watt, B. Ingham, M. F. Toney and R. D. Tilley, J. Am. Chem. Soc., 2009, 131, 14590-14595.

32 X. Teng, X. Liang, S. Maksimuk and H. Yang, Small, 2006, 2, 249-253.

33 C. Wang, H. Daimon, T. Onodera, T. Koda and S. Sun, Angew. Chem., 2008, 120, 3644-3647.

34 Y. Li and Y. Huang, Adv. Mater., 2010, 22, 1921-1925.

35 N. R. Jana and X. Peng, J. Am. Chem. Soc., 2003, 125, 14280-14281.

36 X. Wang, J. Stöver, V. Zielasek, L. Altmann, K. Thiel, K. Al-Shamery, M. Bäumer, H. Borchert, J. Parisi and J. Kolny-Olesiak, Langmuir, 2011, 27, 11052-11061. 